\title{
MSTCN: A multiscale temporal convolutional network for user independent human activity recognition [version 1; peer
} review: 2 approved with reservations]

\author{
Sarmela Raja Sekaran (D1), Ying Han Pang (iD), Goh Fan Ling², Ooi Shih Yin (DiD) \\ ${ }^{1}$ Faculty of Information Science and Technology, Multimedia University, Ayer Keroh, Melaka, 75450, Malaysia \\ ${ }^{2}$ Millapp Sdn Bhd, Bangsar South, Kuala Lumpur, 59200, Malaysia
}

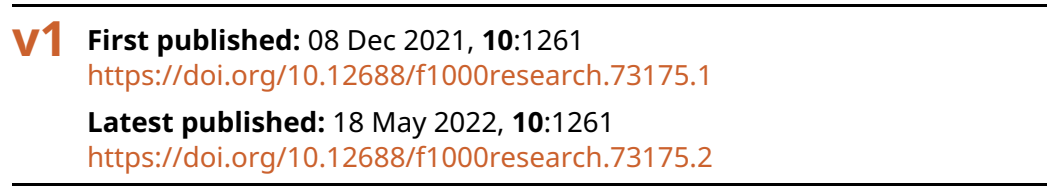

\section{Abstract}

Background: In recent years, human activity recognition (HAR) has been an active research topic due to its widespread application in various fields such as healthcare, sports, patient monitoring, etc. HAR approaches can be categorised as handcrafted feature methods (HCF) and deep learning methods (DL). HCF involves complex data preprocessing and manual feature extraction in which the models may be exposed to high bias and crucial implicit pattern loss. Hence, DL approaches are introduced due to their exceptional recognition performance. Convolutional Neural Network (CNN) extracts spatial features while preserving localisation. However, it hardly captures temporal features. Recurrent Neural Network (RNN) learns temporal features, but it is susceptible to gradient vanishing and suffers from short-term memory problems. Unlike RNN, Long-Short Term Memory network has a relatively longer-term dependency. However, it consumes higher computation and memory because it computes and stores partial results at each level.

Methods: This work proposes a novel multiscale temporal convolutional network (MSTCN) based on the Inception model with a temporal convolutional architecture. Unlike HCF methods, MSTCN requires minimal pre-processing and no manual feature engineering. Further, multiple separable convolutions with different-sized kernels are used in MSTCN for multiscale feature extraction. Dilations are applied to each separable convolution to enlarge the receptive fields without increasing the model parameters. Moreover, residual connections are utilised to prevent information loss and gradient vanishing. These features enable MSTCN to possess a longer effective history while maintaining a relatively low in-network computation. Results: The performance of MSTCN is evaluated on UCI and WISDM datasets using subject independent protocol with no overlapping subjects between the training and testing sets. MSTCN achieves F1 scores of 0.9752 on UCI and 0.9470 on WISDM.

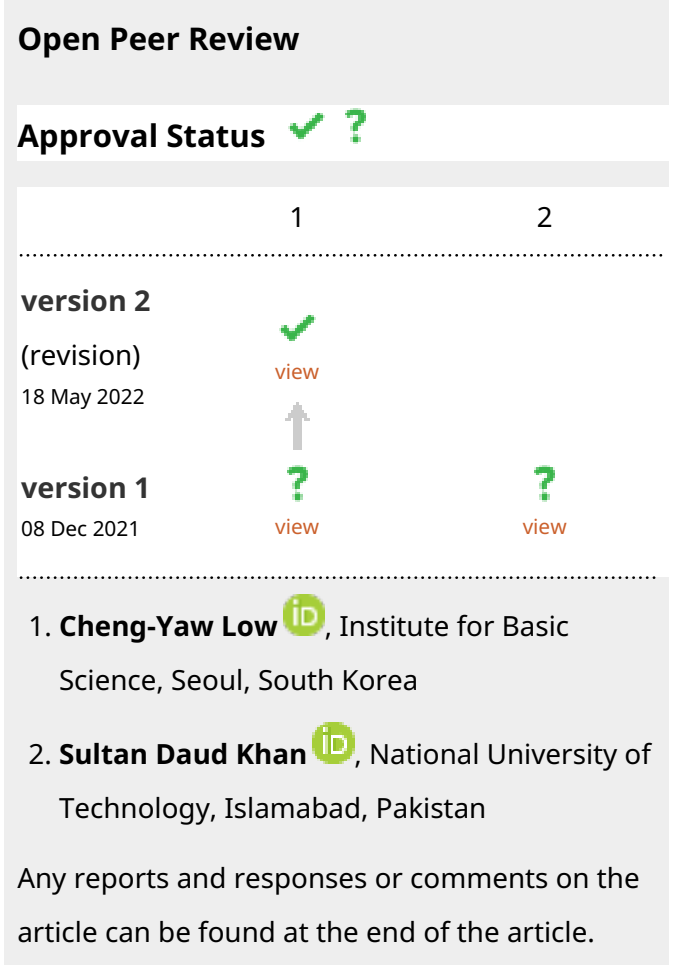


Conclusion: The proposed MSTCN dominates the other state-of-theart methods by acquiring high recognition accuracies without requiring any manual feature engineering.

\section{Keywords}

human activity recognition, smartphone, temporal convolutional

network, dilated convolution, one-dimensional inertial sensor

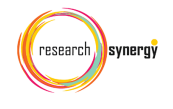

This article is included in the Research Synergy

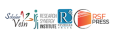

Foundation gateway.

Corresponding author: Ying Han Pang (yhpang@mmu.edu.my)

Author roles: Raja Sekaran S: Conceptualization, Formal Analysis, Investigation, Methodology, Resources, Software, Validation, Visualization, Writing - Original Draft Preparation, Writing - Review \& Editing; Pang YH: Data Curation, Funding Acquisition, Project Administration, Resources, Supervision, Writing - Review \& Editing; Ling GF: Data Curation, Software; Yin OS: Writing - Review \& Editing

Competing interests: No competing interests were disclosed.

Grant information: This study was funded by the Fundamental Research Grant Scheme (FRGS) from the Ministry of Education Malaysia - FRGS/1/2020/ICT02/MMU/02/7. The funder had no role in study design, data collection and analysis, decision to publish, or manuscript preparation.

The funders had no role in study design, data collection and analysis, decision to publish, or preparation of the manuscript.

Copyright: @ 2021 Raja Sekaran S et al. This is an open access article distributed under the terms of the Creative Commons Attribution License, which permits unrestricted use, distribution, and reproduction in any medium, provided the original work is properly cited.

How to cite this article: Raja Sekaran S, Pang YH, Ling GF and Yin OS. MSTCN: A multiscale temporal convolutional network for user independent human activity recognition [version 1; peer review: 2 approved with reservations] F1000Research 2021, 10:1261 https://doi.org/10.12688/f1000research.73175.1

First published: 08 Dec 2021, 10:1261 https://doi.org/10.12688/f1000research.73175.1 


\section{Introduction}

Human activity recognition (HAR) is extensively applied in various applications such as personal health monitoring, ${ }^{1,2}$ geriatric patient monitoring, ${ }^{3}$ ambient assisted living, ${ }^{4}$ etc. The widespread use of smartphone-based HAR is due to the ubiquity of smartphones and low-cost sensors. Additionally, sensor-based HAR provides a non-intrusive solution.

Numerous HAR algorithms have been proposed, including handcrafted feature (HCF) methods ${ }^{5-7}$ and deep learning (DL) methods. ${ }^{8-10}$ HCF methods require complex data pre-processing and manual feature engineering. The manually extracted features are highly dependent on prior knowledge, leading to high bias and loss of essential implicit patterns. Hence, DL methods, such as convolutional neural network $(\mathrm{CNN}){ }^{8,9}$ recurrent neural network (RNN), and long-short term memory network (LSTM), ${ }^{10,11}$ are devised to overcome the downfalls of HCF methods. DL methods involve no complex data pre-processing, and features are automatically tuned for the desired outcome. Besides, the architecture is adaptable to different applications.

Although CNN is good in extracting spatial features, it hardly learns temporal features, which are significant in motion analysis. RNN and LSTM are feasible for time-series data, but they suffer from several shortcomings. For example, RNN is prone to short-term memory problems, leaving out important information at the beginning if the input sequence is too long. LSTM prevails over RNN as the former has a longer-term dependency and is less susceptible to vanishing gradient. However, LSTM requires higher computation due to multiple gate operations and more memory to store partial results throughout the training phase.

This work proposes a multiscale temporal convolutional network, termed MSTCN. As illustrated in Figure 1, MSTCN is constituted by multiscale dilation (MSD) blocks, global average pooling and softmax. The contributions of this work are:

- A deep analytic model, amalgamating the Inception model and Temporal Convolutional Network (TCN), is developed to extract spatial-temporal features from inertial data. MSTCN requires minimal data pre-processing and no manual feature engineering.

- Multiple different-sized convolutions are incorporated in MSTCN to perform multiscale feature extraction. The scaled features encompass low-to-high level features of the data. The concatenation of multiscale features enables MSTCN for better data generalisation.

- Dilated convolution is implemented to improve the convolution kernel's receptive fields. The dilation captures the global characteristics of the inertial data and retains a longer effective history.

- A comprehensive experimental analysis is conducted using two popular public databases, $\mathrm{UCI}^{12}$ and WISDM. ${ }^{13}$ Subject independent protocol is implemented where the training and testing sets do not share the data from the same users.

\section{Related work}

One-dimensional inertial data undergoes a complicated pre-processing in HCF methods to extract salient statistical feature vectors in time and/or frequency domains. The manually extracted features are then fed into standard machine learning classifiers, such as support vector machine (SVM) ${ }^{7,12}$ ADA Boost, ${ }^{14}$ Random Forest, ${ }^{6} \mathrm{C} 4.5$ decision tree, ${ }^{15}$ etc., for activity classification. He and $\mathrm{Jin}^{5}$ proposed a discrete cosine transform method to extract features and classify the features using multiclass SVM. Lara et al., ${ }^{16}$ developed an additive logistic regression, boosting with an ensemble of 10 decision stump classifiers. In the works of Ronao and Cho, ${ }^{17,18}$ the authors explored the Continuous Hidden Markov Model (HMM) to perform activity recognition in two stages, where the first stage is for static and dynamic classification and the second stage is for course classification. Although these methods produce an adequate performance, they are highly dependent on the effectiveness of the manual feature engineering techniques.

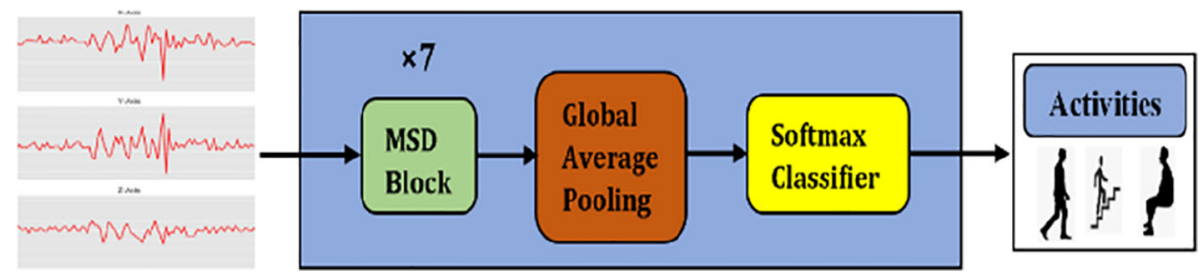

Figure 1. Architecture of MSTCN. 
Recently, researchers leaned towards DL methods since DL requires minimal to zero pre-processing and feature engineering. Ronao et al., ${ }^{8}$ Yazdanbakhsh et al., ${ }^{9}$ and Huang et al., ${ }^{18}$ proposed a CNN-based deep learning system to perform HAR. The reported empirical results show the feasibility of the CNN-based method in analysing motion data. Besides, three-layer LSTM was proposed to classify human activities. ${ }^{11}$ LSTM variant, known as Bidirectional LSTM, was employed in HAR. ${ }^{10}$ This model uses richer information, i.e. previous and subsequent information, to perform activity recognition. Nair et al. proposed two variations of TCN, namely Dilated-TCN and Encoder-Decoder TCN in HAR. ${ }^{19}$ In addition, another two TCN-based models are proposed in Ref. 20, namely TCN-FullyConnectedNetwork and deepConvTCN. Both works of Nair et al., ${ }^{19}$ and Garcia et al., ${ }^{20}$ concluded that the TCN-based models achieved better performance than existing recurrent models due to their longer-term dependencies.

\section{Methods and results}

The raw inertial signals were first pre-processed to remove any null values. Next, the pre-processed signals were segmented using sliding window technique. In specific, the signals were partitioned into fixed-sized time windows and each window did not intersect with another window. Then, the segmented data was fed into seven MSD blocks in MSTCN (green box in Figure 1) for feature extraction. Figure 2 illustrates the structure of an MSD block. MSD block was designed based on the inception module structure for multiple scale feature extraction. ${ }^{21}$

Convolutional unit in MSD block extracts spatial-temporal features of the motion data. The components of the convolutional unit are illustrated in Figure 3. First, the input channels are processed via one-by-one causal convolution for dimensionality reduction. This layer, known as bottleneck layer, adopts fewer filters to reduce the number of features maps while the salient features are retained. The causal padding preserves the input sequence's length and order, preventing information leakage from the future into the past. Next, the reduced feature maps are further processed parallelly by separable convolutions (SepConv) with three different-sized filters to extract features at multiple scales. Figure 4 shows the operation of SepConv. The reason for implementing SepConv in MSTCN is that it can produce fewer parameters and reduce computational complexity.

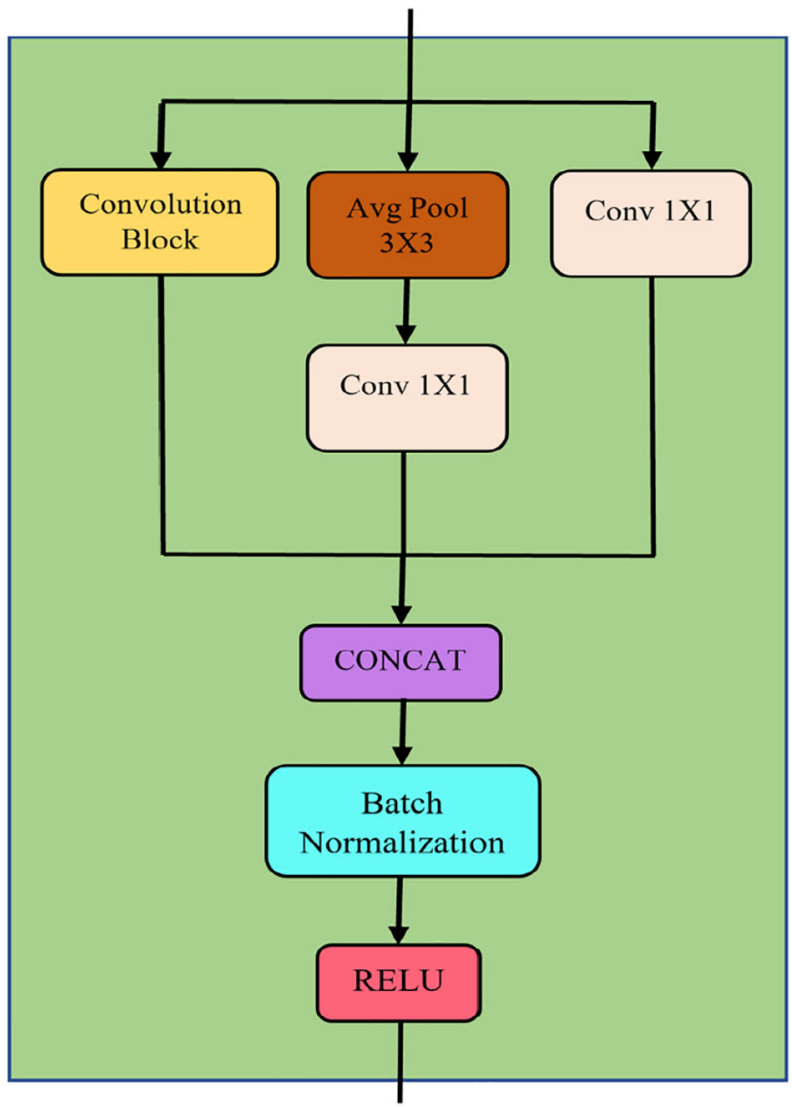

Figure 2. MSD block. 


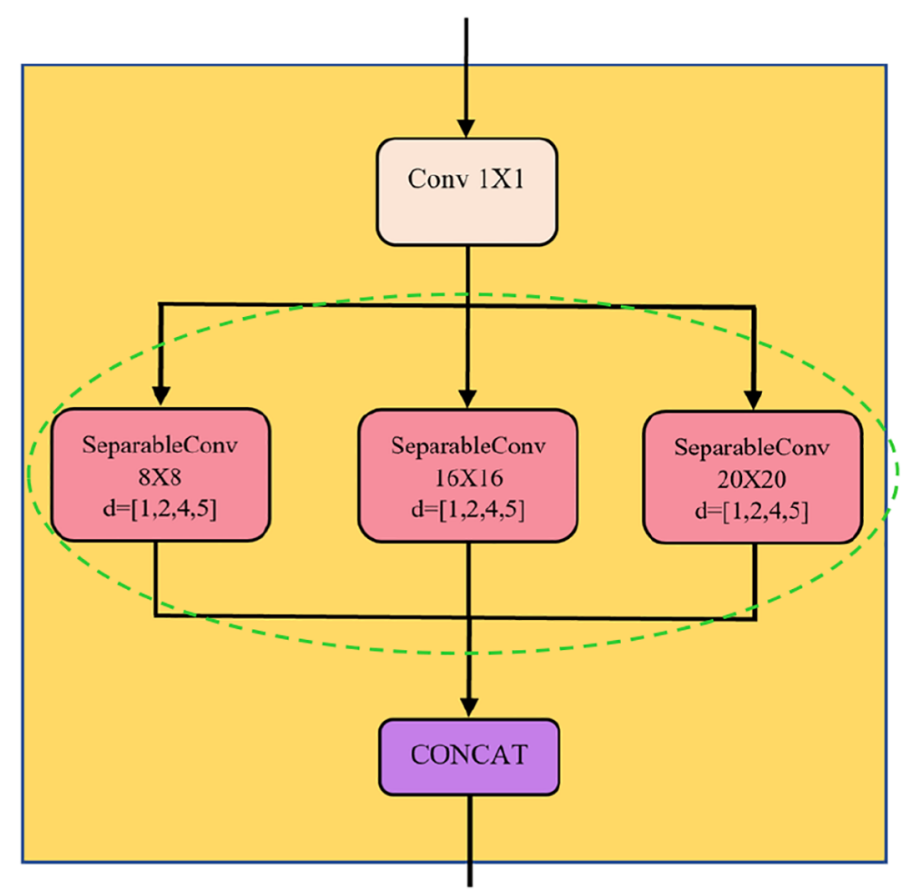

Figure 3. Convolutional unit in MSD block.

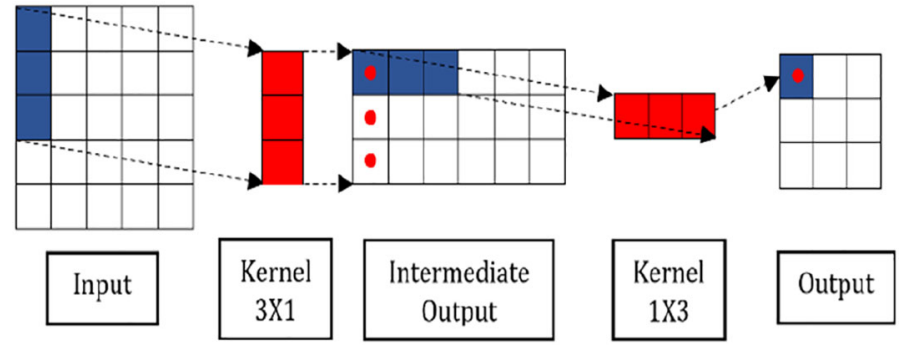

Figure 4. Separable convolution.

Dilated convolution prevails over classical convolution because it allows the model to have a larger receptive field, controlled by the dilation rate. This helps capture long-time sequences' global features without increasing the model's parameters and memory. Figure 5 shows the difference between the dilated convolution and the classical/standard convolution. Dilations are implemented in SepConv to increase the receptive fields of the convolution kernels.

The core difference between MSTCN and TCN is that the dilated convolutions are organised parallelly in MSTCN (green dotted circle in Figure 3) but in a serial form in TCN. With the proposed layout, each extracted multiscale feature from the SepConvs with differently sized filters is concatenated for a better model generalisation, see Figure 3.
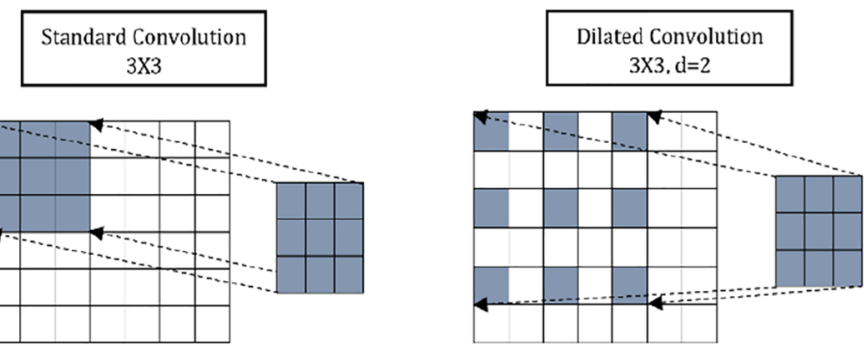

Figure 5. Comparison between normal and dilated convolution. 
In a MSD block, average pooling (brown box in Figure 2) down-samples the feature map to reduce noise and dimensionality. Additionally, it also preserves localisation. The pooling's output is fed into a one-by-one convolution. A residual connection is formed by passing the input into a one-by-one convolution, followed by a batch normalisation. This residual connection ensures longer-term dependencies and prevents information loss. Further, it also reduces the vanishing gradient effects. On the other hand, batch normalisations in MSD block are to reduce the internal covariance shift in the model during training. Furthermore, ReLU activation is chosen for its non-linearity, and the gradient vanishing is minimised.

The features extracted from the series of MSD blocks are further fed into the global average pooling (GAP). In MSTCN, GAP replaces the traditional fully connected layers because GAP is more suitable. ${ }^{22}$ This operation generates one feature map according to each activity from multi-dimensional feature inputs. Besides, GAP is also considered as a structural regulariser since it imposes the generated map as the confidence map for each class. ${ }^{22}$ With this, it better prevents overfitting by reducing the number of model parameters. Additionally, GAP does not require parameter optimisation.

In the classification stage, a simple softmax classifier is used. The softmax activation formula is defined:

$$
\sigma(\vec{z})_{i}=\frac{e^{z_{i}}}{\sum_{j=1}^{K} e^{z_{j}}}
$$

where $\vec{z}$ is the input vector, $e^{z_{i}}$ is the exponential function of the input, $K$ is the number of classes and $e^{z_{j}}$ is the exponential function of the output. This function outputs probabilities of each class, ranging from zero to one, and the target class will have the highest probability.

\section{Experimental setup}

The experiments were conducted on a desktop with Intel ${ }^{\circledR}$ Core $^{\mathrm{TM}}$ i7-8750H CPU with $2.20 \mathrm{GHz}, 16 \mathrm{~GB}$ RAM and NVIDIA GeForce GTX 1050 Ti with Max-Q Design and 4GB memory. Two public databases, UCI ${ }^{12}$ and WISDM, ${ }^{13}$ were used to assess the reliability of the proposed model. In addition, subject independent protocol was implemented where there were no overlapping users between training and testing sets. Details of the databases are recorded in Table 1. The evaluation metrics used in this work include precision, recall, F1 score and classification accuracy.

\section{Experiments}

Three experiments were conducted on UCI dataset to study the effects of (1) convolution, (2) pooling and (3) regularisation on MSTCN's performance. Table 2 shows the proposed model's performances using dilated one-dimensional (1D) causal convolution (CC) and dilated 1D separable convolution (SC). From the empirical results, it was observed that the parameters of SC are approximately half of the parameters of CC. Usually, models with more parameters perform better since maximal data patterns are captured from the training samples. However, when the training sample size is limited, these models might tend to overfit and not generalise properly to the unseen data, leading to poor performance. In this study, SC obtains $\sim 0.04$ higher F1 score than CC.

Next, the performances of max-pooling and average pooling were studied. From Table 3, average pooling dominates max-pooling by attaining F1 score of 0.9752 . Average pooling performs better in this domain because it takes every value into account. With this, the information leakage is prevented, and feature localisation is preserved.

Table 1. Description of UCI and WISDM datasets.

\begin{tabular}{|l|l|l|}
\hline & UCI & WISDM \\
\hline Sensor & Accelerometer and Gyroscope & Accelerometer \\
\hline Segment size & 128 & 128 \\
\hline Segment interval & 50 & 20 \\
\hline Channel size & 9 & 3 \\
\hline Training testing split & 21 training users: 9 testing users & 31 training users: 5 testing users \\
\hline Validation split & $10 \%$ of the training set & $10 \%$ of the training set \\
\hline
\end{tabular}


Table 2. Performance of MSTCN using different convolutions.

\begin{tabular}{|l|l|l|}
\hline & $\begin{array}{l}\text { Dilated 1D } \\
\text { causal convolution }\end{array}$ & $\begin{array}{l}\text { Dilated 1D } \\
\text { separable convolution }\end{array}$ \\
\hline Number of parameters & 6062086 & 3750406 \\
\hline Precision & 0.9357 & $\mathbf{0 . 9 7 6 1}$ \\
\hline Recall & 0.9375 & $\mathbf{0 . 9 7 5 0}$ \\
\hline F1 score & 0.9356 & $\mathbf{0 . 9 7 5 2}$ \\
\hline Accuracy & 93.62 & $\mathbf{9 7 . 4 6}$ \\
\hline
\end{tabular}

Table 3. Performance of MSTCN using different pooling layers.

\begin{tabular}{|l|l|l|}
\hline & Max pooling & Average pooling \\
\hline Precision & 0.9478 & $\mathbf{0 . 9 7 6 1}$ \\
\hline Recall & 0.9468 & $\mathbf{0 . 9 7 5 0}$ \\
\hline F1 score & 0.9463 & $\mathbf{0 . 9 7 5 2}$ \\
\hline Accuracy & 94.67 & $\mathbf{9 7 . 4 6}$ \\
\hline
\end{tabular}

Table 4. Performance of MSTCN using different regularisation settings.

\begin{tabular}{|l|l|l|l|l|}
\hline & L1 & L2 & L1 and L2 & Without regularisation \\
\hline Precision & 0.9485 & 0.9666 & $\mathbf{0 . 9 7 6 1}$ & 0.9529 \\
\hline Recall & 0.9464 & 0.9650 & $\mathbf{0 . 9 7 5 0}$ & 0.9521 \\
\hline F1 score & 0.9459 & 0.9649 & $\mathbf{0 . 9 7 5 2}$ & 0.9517 \\
\hline Accuracy & 94.60 & 96.44 & $\mathbf{9 7 . 4 6}$ & 95.28 \\
\hline
\end{tabular}

Table 5. Accuracy for user independent UCI dataset.

\begin{tabular}{|c|c|c|}
\hline & Type & Accuracy (\%) \\
\hline Statistical features + SVM ${ }^{12}$ & $\mathrm{HCF}$ & 96.00 \\
\hline Statistical features + Continuous $\mathrm{HMM}^{17}$ & $\mathrm{HCF}$ & 91.76 \\
\hline Statistical features + HMM Ensemble ${ }^{23}$ & $\mathrm{HCF}$ & 83.51 \\
\hline Statistical features + $\mathrm{RF}^{24}$ & $\mathrm{HCF}$ & 78.00 \\
\hline Statistical features + Linear SVM ${ }^{7}$ & $\mathrm{HCF}$ & 86.00 \\
\hline Statistical features + Hierarchical Continuous $\mathrm{HMM}^{25}$ & $\mathrm{HCF}$ & 93.18 \\
\hline Statistical features + Dropout Classifiers ${ }^{24}$ & $\mathrm{DL}$ & $\sim 76.00$ \\
\hline Statistical features + Data Centering $+\mathrm{CNN}^{26}$ & $\mathrm{DL}$ & 97.63 \\
\hline $\mathrm{CNN}^{8}$ & $\mathrm{DL}$ & 94.79 \\
\hline Frequency features $+\mathrm{CNN}^{8}$ & $\mathrm{DL}$ & 95.75 \\
\hline Bidirectional LSTM ${ }^{10}$ & $\mathrm{DL}$ & 93.79 \\
\hline Dilated TCN ${ }^{19}$ & $\mathrm{DL}$ & 93.80 \\
\hline Encoder-Decoder TCN ${ }^{19}$ & $\mathrm{DL}$ & 94.60 \\
\hline Statistical features + MLP 27 & DL & 95.00 \\
\hline Frequency and Power features + Multichannel $\mathrm{CNN}^{28}$ & DL & 95.25 \\
\hline Statistical features + InnoHAR ${ }^{29}$ & $\mathrm{DL}$ & 94.50 \\
\hline MSTCN (Proposed Method) & DL & 97.46 \\
\hline
\end{tabular}


Table 6. Accuracy for user independent WISDM dataset.

\begin{tabular}{|c|c|c|}
\hline Methods & Type & Accuracy (\%) \\
\hline Statistical features $+\mathrm{RF}^{24}$ & $\mathrm{HCF}$ & 83.46 \\
\hline Statistical features $+\mathrm{RF}^{6}$ & $\mathrm{HCF}$ & 83.35 \\
\hline Statistical features + Dropout Classifiers ${ }^{24}$ & $\mathrm{DL}$ & 85.36 \\
\hline Statistical features $+\mathrm{CNN}^{26}$ & $\mathrm{DL}$ & 93.32 \\
\hline Dilated and Strided $\mathrm{CNN}^{9}$ & $\mathrm{DL}$ & 88.27 \\
\hline Data Augmentation + Two Stage End-to-End CNN ${ }^{18}$ & $\mathrm{DL}$ & 84.60 \\
\hline Statistical features $+\mathrm{CNN}^{30}$ & $\mathrm{DL}$ & 94.18 \\
\hline MSTCN (Proposed Method) & $\mathrm{DL}$ & 95.20 \\
\hline
\end{tabular}

Table 4 shows the performance of MSTCN with different regularisation settings. The regularisation is performed at the bottleneck layer in MSTCN. L1 is good at dealing with outliers and sparse feature spaces. Moreover, it also reduces the coefficient of the insignificant features to zero and removes them. It is a good feature selector. L2 learns complex patterns from the dataset and prevents overfitting. By combining the usage of L1 and L2, we can leverage the benefits from both. Hence, the best result of $97.5 \%$ accuracy is obtained with L1 and L2 regularisation.

\section{Comparison with other state-of-the-art methods}

A performance comparison between MSTCN and other state-of-the-art methods was conducted. Tables 5 and 6 show the performance on UCI and WISDM datasets using subject independent protocol. The proposed MSTCN showed extraordinary performances against the existing methods by achieving 97.46\% accuracy on UCI and 95.20\% on WISDM. The experimental results will be discussed further in the following section.

\section{Discussion}

From the empirical results, we observe that:

1) MSTCN prevails over HCF methods on both datasets because the proposed model can better capture discriminating features from the motion data. Unlike handcrafted features, these deep features are less biased as they are not dependent on prior knowledge. This is crucial, especially for a subject independent solution.

2) Generally, MSTCN outperforms most CNN-based approaches, with accuracy scores of $\sim 97.5 \%$ in UCI and 95.2\% in WISDM. This performance exhibits that MSTCN can capture the global and local features that discriminate each activity. Besides, the implementation of GAP in MSTCN is less prone to overfitting. ${ }^{22}$ Hence, it is suitable for subject independent HAR.

3) MSTCN dominates the recurrent model ${ }^{10}$ due to its ability in modelling longer-term dependencies via dilated convolution. Residual connection and ReLU activation in MSTCN allow the model to be less susceptible to gradient vanishing and exploding.

4) MSTCN is a TCN-variant model. The obtained empirical results demonstrate that MSTCN outperforms the ordinary TCNs (Dilated TCN and Encoder-Decoder TCN). ${ }^{19}$ MSTCN learns features at multiple scales via different convolutions with differently sized filters. The concatenation of these multi-scaled features produces global feature maps encompassing each activity class low-to-high level features, leading to better recognition.

\section{Conclusions}

A new deep analytic model, known as MSTCN, is proposed for subject independent HAR. MSTCN is based on the architectures of the Inception network and temporal convolutional network. In MSTCN, different-sized filters are adopted in dilated separable convolutions to extract multiscale features with the enlarged receptive field of each kernel for longer-term dependencies modelling. Besides, average pooling is performed for dimensionality reduction and locality preservation. The inclusion of residual connections in MSTCN prevents information leakage throughout the network. The efficiency of MSTCN is evaluated using UCI and WISDM datasets. The empirical results demonstrate the superiority of MSTCN over other state-of-the-art solutions by achieving 0.9752 and $0.9470 \mathrm{~F} 1$ scores, respectively, in UCI and WISDM. 
Data availability

All data underlying the results are available as part of the article and no additional source data are required.

References

1. Li $H$, Trocan M: Deep learning of smartphone sensor data for personal health assistance. Microelectronics J. 2019; 88(January 2018): 164-172.

Publisher Full Text

2. Yang $S$, et al.: IoT structured long-term wearable social sensing for mental wellbeing. IEEE Internet Things J. Apr. 2019; 6(2): 3652-3662.

Publisher Full Text

3. Chen $X$, Xue H, Kim M, et al.: Detection of Falls with Smartphone Using Machine Learning Technique. Proceedings - 2019 8th International Congress on Advanced Applied Informatics, IIAI-AAI 2019. 2019; pp. 611-616.

4. Wan J, Li M, O'Grady MJ, et al.: Time-Bounded Activity Recognition for Ambient Assisted Living. IEEE Trans. Emerg. Top. Comput. Jan. 2021; 9(1): 471-483.

Publisher Full Text

5. He Z, Jin L: Activity recognition from acceleration data based on discrete consine transform and SVM. Conference Proceedings - IEEE International Conference on Systems, Man and Cybernetics. 2009; pp. 5041-5044.

6. Kee YJ, Shah Zainudin MN, Idris MI, et al.: Activity recognition on subject independent using machine learning. Cybern. Inf. Technol. Sep. 2020; 20(3): 64-74.

7. Seto $S$, Zhang W, Zhou Y: Multivariate time series classification using dynamic time warping template selection for human activity recognition. Proceedings - 2015 IEEE Symposium Series on Computational Intelligence, SSCI 2015. 2015; 1399-1406.

8. Ronao CA, Cho SB: Human activity recognition with smartphone sensors using deep learning neural networks. Expert Syst. Appl. Oct. 2016; 59: 235-244

Publisher Full Text

9. Yazdanbakhsh O, Dick S: Multivariate Time Series Classification using Dilated Convolutional Neural Network. arXiv. 2019.

10. Yu S, Qin L: Human activity recognition with smartphone inertial sensors using bidir-LSTM networks. Proc. - 2018 3rd Int. Conf. Mech. Control Comput. Eng. ICMCCE 2018. 2018; pp. 219-224.

11. Pienaar SW, Malekian R: Human Activity Recognition using LSTMRNN Deep Neural Network Architecture. 2019 IEEE 2nd Wireless Africa Conference, WAC 2019 - Proceedings. 2019.

12. Anguita $D$, Ghio $A$, Oneto $L$, et al.: A public domain dataset for human activity recognition using smartphones. ESANN 2013 proceedings, 21st European Symposium on Artificial Neural Networks, Computational Intelligence and Machine Learning. 2013. [Accessed: 17-Sep-2021]

Reference Source

13. Kwapisz JR, Weiss GM, Moore SA: Activity recognition using cell phone accelerometers. ACM SIGKDD Explor. NewsI. 2011; 12(2): 74-82. Publisher Full Text

14. Kumar A, Gupta S: Human Activity Recognition through Smartphone's Tri-Axial Accelerometer using Time Domain Wave Analysis and Machine Learning Simulation and Application performance evaluation using GPU through CUDA C \& Deep Learning in TensorFlow View project Human Activi. Artic. Int. J. Comput. Appl. 2015; 127(18): 22-26. Publisher Full Text

15. Anjum A, Ilyas MU: Activity recognition using smartphone sensors. 2013 IEEE 10th Consumer Communications and Networking Conference, CCNC 2013. 2013; pp. 914-919.
16. Lara ÓD, Prez AJ, Labrador MA, et al.: Centinela: A human activity recognition system based on acceleration and vital sign data. Pervasive and Mobile Computing.. 2012; 8(5): 717-729. Publisher Full Text

17. Ronao $\mathrm{CA}, \mathrm{Cho} \mathrm{SB}$ : Human activity recognition using smartphone sensors with two-stage continuous hidden markov models. 2014 10th International Conference on Natural Computation, ICNC 2014. 2014; pp. 681-686.

18. Huang J, Lin S, Wang N, et al.: TSE-CNN: A Two-Stage End-to-End CNN for Human Activity Recognition. IEEE J. Biomed. Heal. Informatics. Jan. 2020; 24(1): 292-299. Publisher Full Text

19. Nair $\mathrm{N}$, Thomas C, Jayagopi DB: Human activity recognition using temporal convolutional network. ACM Int. Conf. Proceeding Ser. 2018.

20. Garcia FA, Ranieri CM, Romero RAF: Temporal approaches for human activity recognition using inertial sensors. Proc. - 2019 Lat. Am. Robot. Symp. 2019 Brazilian Symp. Robot. 2019 Work. Robot Educ. LARS/SBR/WRE 2019. 2019; pp. 121-125.

21. Szegedy $C$, et al.: Going deeper with convolutions. Proceedings of the IEEE Computer Society Conference on Computer Vision and Pattern Recognition. 2015; 07-12-June: 1-9.

22. Lin $\mathrm{M}$, Chen $\mathrm{Q}$, Yan S: Network in network. 2nd International Conference on Learning Representations, ICLR 2014 - Conference Track Proceedings. 2014.

23. Kim YJ, Kang BN, Kim D: Hidden Markov Model Ensemble for Activity Recognition Using Tri-Axis Accelerometer. Proceedings 2015 IEEE International Conference on Systems, Man, and Cybernetics, SMC 2015. 2016; 3036-3041.

24. Kolosnjaji B, Eckert C: Neural network-based user-independent physical activity recognition for mobile devices. Lecture Notes in Computer Science (including subseries Lecture Notes in Artificial Intelligence and Lecture Notes in Bioinformatics). 2015; vol. 9375 LNCS: pp. 378-386.

25. Ronao $\mathrm{CA}$, Cho $\mathrm{SB}$ : Recognizing human activities from smartphone sensors using hierarchical continuous hidden Markov models. Int. J. Distrib. Sens. Networks. 2017; 13(1): 155014771668368. Publisher Full Text

26. Ignatov A: Real-time human activity recognition from accelerometer data using Convolutional Neural Networks. Appl. Soft Comput. J. 2018; 62: 915-922. Publisher Full Text

27. Ogbuabor G, La R: Human activity recognition for healthcare using smartphones. ACM Int. Conf. Proceeding Ser. 2018; 41-46.

28. Sikder N, Chowdhury MS, Arif ASM, et al.: Human activity recognition using multichannel convolutional neural network. 2019 5th International Conference on Advances in Electrical Engineering, ICAEE 2019. 2019; pp. 560-565.

29. Xu C, Chai D, He J, et al.: InnoHAR: A deep neural network for complex human activity recognition. IEEE Access. 2019; 7: 9893-9902. Publisher Full Text

30. Peppas K, Tsolakis AC, Krinidis S, et al.: Real-time physical activity recognition on smart mobile devices using convolutional neural networks. Appl. Sci. 2020; 10(23): 1-25.

Publisher Full Text 


\section{Open Peer Review}

\section{Current Peer Review Status: ? ?}

\section{Version 1}

Reviewer Report 02 February 2022

https://doi.org/10.5256/f1000research.76807.r102276

(C) 2022 Khan S. This is an open access peer review report distributed under the terms of the Creative Commons Attribution License, which permits unrestricted use, distribution, and reproduction in any medium, provided the original work is properly cited.

\section{Sultan Daud Khan}

Department of Computer Science, National University of Technology, Islamabad, Pakistan

In this work, the authors proposed a framework for human activities recognition. The authors proposed a multi-scale temporal convolutional network that constituted multi-scale dilations block to capture multi-scale information. Overall, the paper is not well-written and organized and I have the following concerns the authors need to consider:

1. The contribution of the work is not clear. As there is a lot of literature on human activity recognition systems. How is the proposed framework different from its counterparts?

2. What are the gaps the authors are trying to fill which are left behind by the previous approaches?

3. Figure-1 should be improved, and more details should be incorporated.

4. Discussion section should not be in the bullets form. Please write detail in paragraph.

5. The authors should perform comparison with the following reference:

"Stacked Istm network for human activity recognition using smartphone data." In 2019 8th European workshop on visual information processing (EUVIP), pp. 175-180. IEEE, 2019.

\section{References}

1. Ullah m, Ullah H, Khan SD, Cheikh FA: Stacked Lstm Network for Human Activity Recognition Using Smartphone Data. 2019 8th European Workshop on Visual Information Processing (EUVIP). 2019. 175-180 Publisher Full Text

Is the work clearly and accurately presented and does it cite the current literature? No

Is the study design appropriate and is the work technically sound? 
Partly

Are sufficient details of methods and analysis provided to allow replication by others? No

If applicable, is the statistical analysis and its interpretation appropriate?

Partly

Are all the source data underlying the results available to ensure full reproducibility?

Yes

Are the conclusions drawn adequately supported by the results?

No

Competing Interests: No competing interests were disclosed.

Reviewer Expertise: Computer Vision

I confirm that I have read this submission and believe that I have an appropriate level of expertise to confirm that it is of an acceptable scientific standard, however I have significant reservations, as outlined above.

Author Response 07 May 2022

Sarmela Raja Sekaran

First of all, we would like to convey our heartfelt thanks to the Editors and Reviewers who have provided us with constructive comments which allowed us to improve our work.

1. (a) The contribution of the work is not clear. As there is a lot of literature on human activity recognition systems.

Response: Thanks for the feedback. The authors have revised the contribution of the work for better clarification.

(b)How is the proposed framework different from its counterparts?

Response: Thanks for the comment. For better clarification, the authors have revised the part of Contribution in the section Introduction to include the difference between the proposed model and the existing methods:

Unlike the existing methods, the proposed method does not require either complex signal pre-processing or manual feature engineering by experts. Besides, the proposed MSTCN is capable of extracting features at multiple scales and concatenating them for a better representation of the overall features. Additionally, the adoption of the dilated convolutions enables the proposed model to preserve longer-term dependencies.

2. What are the gaps the authors are trying to fill which are left behind by the previous 
approaches?

Response: Authors have revised the Introduction section and included the research gap in HAR.

3. Figure-1 should be improved, and more details should be incorporated.

Response: Authors have revised Figure 1 and included more details for better clarification.

4. Discussion section should not be in the bullets form. Please write detail in paragraph.

Response: Authors have revised the Discussion section. The discussion was written in paragraph form.

5. The authors should perform comparison with the following reference:"Stacked Istm network for human activity recognition using smartphone data." In 2019 8th European workshop on visual information processing (EUVIP), pp. 175-180. IEEE, 2019.

Response: Thanks for the suggestion. Authors have included the comparison with the suggested reference in Related Work section and the Experiments section.

Competing Interests: No competing interests were disclosed.

Reviewer Report 10 January 2022

https://doi.org/10.5256/f1000research.76807.r102273

(C) 2022 Low C. This is an open access peer review report distributed under the terms of the Creative Commons Attribution License, which permits unrestricted use, distribution, and reproduction in any medium, provided the original work is properly cited.

\section{Cheng-Yaw Low}

Institute for Basic Science, Seoul, South Korea

This is a poorly written manuscript, as many sections are unclear and most of the important information is missing.

1. The Inception model is recruited as the network backbone without any justifications. To be specific, why is Inception model useful for HAR?

2. The methodology section is difficult to read as it is incomplete. For example, the input dimension is unknown? and therefore I do not know why $1 \times 1$ conv is demanded for dimensionality reduction?

"First, the input channels are processed via one-by-one causal convolution for dimensionality reduction." 
For clarity, the basic mathematical representation elaborating each operation should be included. On the other hand, I think the methods and results section should be separated into two.

3. Figure captions contain no detail at all, and this makes the reading very difficult. For example, the authors do not provide in Fig. 3 (and the entire manuscript) the definition for $\mathrm{d}=[1,2,4,5]$ ? What are represented by $8 \times 8,16 \times 16,20 \times 20$ ? How was the feature concatenation is performed? By an arithmetic operation? Or stacking over different feature tensors?

4. There are a number of ambiguous or misleading statements throughout the manuscript.

(a) In MSTCN, GAP replaces the traditional fully connected layers because GAP is more suitable. This operation generates one feature map according to each activity from multidimensional feature inputs.

>> GAP generates one feature map according to each activity?

(b) First, the input channels are processed via one-by-one causal convolution for dimensionality reduction. This layer, known as bottleneck layer, adopts fewer filters to reduce the number of features maps while the salient features are retained.

>> The causal convolutional layer is not a bottleneck layer.

(c) Subject independent protocol is implemented where the training and testing sets do not share the data from the same users.

>> The training and the testing sets do not share the data from the same users always. I think the authors are claiming that the training and testing identities (instead of data) are disjoint. State also why this training protocol is important in HAR.

(d) L2 learns complex patterns from the dataset and prevents overfitting.

>> L2 is only a normalization technique, and L2 does not learn.

(e) The pooling's output is fed into a one-by-one convolution. A residual connection is formed by passing the input into a one-by-one convolution, followed by a batch normalisation.

>> There is no residual connection found from both Fig. 2 or 3 ?

>> Dilated convolution captures global features? What are these global features? and by how the global features are captured?

5. This manuscript contains only ONE mathematical equation, but it is problematic. In the meantime, the definitions for each variable should also be provided, e.g., what is z_i? what is meant by "simple" softmax classifier? Cross-entropy? 
6. The dataset information, the training procedures and the empirical hyperparameters are not disclosed?

(a) What is the input dimension for each dataset? What is the data captured by accelerometer and gyroscope? What are the class number for each dataset?

(b) What is feature dimension rendered by the MSTCN? and from which layer the feature representation is extracted for inference purposes?

(c) The optimizer, learning rate, weight decay, batch size, etc., are unknown.

(d) The measurement unit for segment size and segment interval in Table 1 should be indicated.

Is the work clearly and accurately presented and does it cite the current literature? Partly

Is the study design appropriate and is the work technically sound? Partly

Are sufficient details of methods and analysis provided to allow replication by others? No

If applicable, is the statistical analysis and its interpretation appropriate? Partly

Are all the source data underlying the results available to ensure full reproducibility? No

Are the conclusions drawn adequately supported by the results? Partly

Competing Interests: No competing interests were disclosed.

Reviewer Expertise: Deep Learning, Computer Vision, Pattern Recognition, Biometric Recognition.

I confirm that I have read this submission and believe that I have an appropriate level of expertise to confirm that it is of an acceptable scientific standard, however I have significant reservations, as outlined above.

Author Response 07 May 2022

Sarmela Raja Sekaran

First of all, we would like to convey our heartfelt thanks to the Editors and Reviewers who have provided us with the constructive comments which allowed us to improve our work. 
1. The Inception model is recruited as the network backbone without any justifications. To be specific, why is the Inception model useful for HAR?

Response: Thanks for the feedback. The main reason for recruiting the Inception model as the network backbone is because the Inception model allows multiscale feature extraction. The authors have revised the Methods section to include the justification of recruiting the Inception model for better clarification.

2. (a) The methodology section is difficult to read as it is incomplete. For example, the input dimension is unknown? and therefore I do not know why $1 \times 1$ Conv is demanded dimensionality reduction?

Response: The Methods section has been revised for better clarification. In the revised version, the authors have included the input dimension in the Model configuration and experimental setup section. The reason for employing 1x1 Conv has also been included in the Method section.

(b) I think the methods and results section should be separated into two.

Response: Authors have separated the Methods and Results sections as suggested by the reviewer.

3. (a) Figure captions contain no detail at all, and this makes the reading very difficult. For example, the authors do not provide in Fig. 3 (and the entire manuscript) the definition for $d=[1,2,4,5]$ ? What is represented by $8 \times 8,16 \times 16$, and $20 \times 20$ ?

Response: Authors have modified Figure 3 and provided a clearer explanation of the figure in the Methods section.

(b) How was the feature concatenation is performed? By an arithmetic operation? Or stacking over different feature tensors?

Response: Feature concatenation is performed by stacking over different feature maps. The Methods section has been revised to include the information for better clarification.

4. There are a number of ambiguous or misleading statements throughout the manuscript (a) GAP generates one feature map according to each activity?

Response: Authors have revised the explanation in the Methods section for better clarification.

(b) The causal convolutional layer is not a bottleneck layer.

Response: Authors have revised the explanation in the Methods section for better clarification. 
(c) The training and the testing sets do not share the data from the same users always. I think the authors are claiming that the training and testing identities (instead of data) are disjoint. State also why this training protocol is important in HAR

Response: For better clarification, the section on Model Configuration and Experimental Setup has been revised. The subject independent protocol is adopted in this work. In other words, there are no overlapping users between training and testing sets. This protocol is preferable for real-time HAR applications.

(d) L2 learns complex patterns from the dataset and prevents overfitting.

Response: Authors have revised the explanation in the Experiments section for better clarification.

(e) There is no residual connection found from both Fig. 2 or 3 ?

Response: Authors have revised Figure 2 and included more information regarding residual connection in the Methods section.

(f) Dilated convolution captures global features? What are these global features? and by how the global features are captured?

Response: Authors have revised the Methods section for better clarification. Confusing sentences have been revised. Dilated convolution enables longer-term time dependency of the proposed model by enlarging the convolution's receptive fields.

5. This manuscript contains only ONE mathematical equation, but it is problematic. In the meantime, the definitions for each variable should also be provided, e.g., what is zi? what is meant by "simple" softmax classifier? Cross-entropy?

Response: Authors have revised and included equations (softmax activation and crossentropy functions) as well as the definition for each variable of each equation in the Methods section for better clarification. Softmax classifier is implemented in the proposed model for classification purposes. Further, the categorical cross-entropy loss has been implemented to the softmax function output.

6. The dataset information, the training procedures and the empirical hyperparameters are not disclosed??

(a) What is the input dimension for each dataset? What is the data captured by the accelerometer and gyroscope? What is the class number for each dataset?

Response: Authors have included detailed information about the datasets including input dimension, class number and data type in the Experiments and Results section.

(b) What is the feature dimension rendered by the MSTCN? and from which layer the feature representation is extracted for inference purposes? 
Response: Softmax classifier is implemented for inference purposes. The authors have revised the Methods section for better clarification.

(c) The optimizer, learning rate, weight decay, batch size, etc., are unknown

Response: Authors have included information regarding optimizer, learning rate, batch size, etc., in the Model configuration and experimental setup section for better clarification.

(d) The measurement unit for segment size and segment interval in Table 1 should be indicated.

Response: Authors have provided the measurement unit for segment size and segment interval in the Model configuration and experimental setup section.

Competing Interests: No competing interests were disclosed.

The benefits of publishing with F1000Research:

- Your article is published within days, with no editorial bias

- You can publish traditional articles, null/negative results, case reports, data notes and more

- The peer review process is transparent and collaborative

- Your article is indexed in PubMed after passing peer review

- Dedicated customer support at every stage

For pre-submission enquiries, contact research@f1000.com 